评述中国知名大学及研究院所专栏 中国科学院烟台海岸带研究所专辑

\title{
仿生防污技术研究进展与展望
}

\author{
王建华，杨翠云*，刘苏静，方圣涛，夏传海* \\ 中国科学院烟台海岸带研究所, 海岸带生物学与生物资源利用重点实验室, 烟台 264003 \\ * 联系人, E-mail: cyyang@yic.ac.cn; chxia@yic.ac.cn
}

收稿日期: 2016-01-22; 接受日期: 2016-02-16; 网络版发表日期: 2016-08-26

国家自然科学基金(批准号: 41206154, 21202198)资助

\begin{abstract}
摘要 海洋生物污损问题给海洋事业的发展带来了许多危害, 传统防污技术已日渐不能满足要求, 研发新型 环境友好型防污剂迫在眉睫. 用仿生学原理和化学生态学的方法发展新型无毒仿生防污材料和技术是解决海洋 污损问题的新思路. 本文综述了污损生物防除技术的发展, 并重点介绍了基于化学生态学发现的仿生抗生物附 着先导化合物和防污材料, 展望了仿生防污技术的发展趋势.
\end{abstract}

关键词生物污损, 防污剂, 化学生态学, 仿生防污技术

海洋生物污损是指海水中的人工设施表面上, 海洋污损生物的大量聚集生长和繁殖现象 ${ }^{[1]}$. 海洋生 物污损在全球范围内造成巨大的经济损失，也是人 类利用和开发海洋资源过程中难以解决的问题. 生 物污损不仅加速金属的腐蚀、增加燃料消耗, 而且影 响水产养殖业的产量和质量, 由此造成的全球经济 损失高达 400 500 亿美元/年 ${ }^{[1]}$. 因此, 人们积极采用 各种物理、化学、生物的方法来进行生物污损的防除, 如机械清除、电解水、超声、涂装防污涂料等 ${ }^{[2]}$, 其 中涂装防污涂料是目前最有效的一种防污方法. 传 统防污剂由于自身的环境毒性给海洋生物带来了巨 大危害, 国际海事组织决定, 至 2010 年在全球范围 内禁止使用以有机金属为主要成分的防污漆. 因此, 寻找一种有效的、环境友好型的防污剂成了人类迫在 眉睫的重要问题.

\section{1 海洋生物污损及其危害}

海洋占地球面积的 $70 \%$ 以上, 蕴藏着丰富的生 物资源. 人类自开发利用海洋资源以来, 一直为海洋 生物附着所引起的污损问题所困扰. 海洋污损生物 或称海洋附着生物是指长期附着在海上人工设施上, 并对人类的海洋经济活动带来巨大损失的海洋动物、 植物和微生物的总称. 由于它们的附着而对设施所 造成的种种危害, 统称为海洋生物污损. 据统计海洋 中约有 5000 多种污损生物, 隶属于海洋菌类、藻类 以及海洋动物等各个主要门类. 除微生物外, 约有 600 种植物性生物和 1300 种动物性生物, 其中 $1 / 2$ 以 上生活在海岸及港湾地区. 在中国沿岸已经记录的 有 650 种左右的污损生物, 常见的有吘苔、石莼、水 云、藤壶、贻贝、牡蚛、水螅、苔藓虫、海绵、海鞘 等. 海洋污损生物附着的一般过程是有机碎屑等最

引用格式: 王建华, 杨翠云, 刘苏静, 等. 仿生防污技术研究进展与展望. 中国科学: 生命科学, 2016, 46: 1079-1084

Wang J H, Xia C H, Yang C Y, et al. Novel antifouling technology research: progress and prospects. Sci Sin Vitae, 2016, 46: 1079-1084, doi: $10.1360 /$ N052016-00065 
先富集黏附, 然后细菌、硅藻等微型污损生物附着生 长形成生物膜, 最后大型藻类、苔藓虫、藤壶、贻贝 等大型污损生物的孢子、幼体附着、变态生长 ${ }^{[3]}$. 在 生物污损形成过程中经历了由少到多、个体由小到 大、由个体到群落的发展过程. 具体过程如图 1 所示.

海洋污损生物的附着给海防、航运、港工建筑、 水产业、海洋石油开发等海洋事业带来巨大危害. 美 国海军研究室称, 微型污损生物所形成污损生物膜 会给舰船航行增加高达 $20 \%$ 的阻力, 而由藤壶、贻贝 等大型污损生物造成的大面积污损则能将航行阻力 增大 $60 \%$ 以上, 美国海上作战中心推测这将使船的 航行速度下降 10\%, 船舶燃料消耗增加 30\%以上, 海 军为应对海洋生物污损每年要耗费约 10 亿美元 ${ }^{[4]}$. 在海水养殖业方面, 生物污损可导致鱼箱、扇贝笼的 堵塞等, 从而导致所养殖的海洋生物因缺氧而死亡, 由此造成的经济损失每年可达 30 50 亿美元. 此外, 海洋污损生物对沿海发电厂的抽水管道、交换膜、热 交换器等的污损, 每年造成的损失可达 160 亿美元. 据不完全统计, 每年全世界由海洋生物污损造成的 直接损失和消耗达 400 500 亿美元 ${ }^{[4]}$. 著名的海洋化 学家 Horne 先生 ${ }^{[5]}$ 评价说: 自古以来海洋生物的污损 比起腐蚀来是个更为麻烦的问题, 污损生物生命力 坚韧, 使得污损问题成为人类征服海洋的一个难以 逾越的障碍.

\section{2 海洋生物污损防除技术}

人类同海洋污损生物的斗争已有两千年的历史, 为了防止被海洋生物附着, 人们积极采用了各种方 法来进行生物污损的防除. 最简单的方法如利用物 理手段并借助必要的人力和物力等外在条件来清除
设施等结构物表面的污损生物, 从而达到污损防除 的目的 ${ }^{[6]}$. 根据其所采用的清除手段的不同, 可分为 机械清除法、外加电流法、超声波法防污法、辐射法 等. 另外还有利用某些化学品的毒性将污损生物毒 杀或灭活, 从而干扰污损生物的附着, 包括直接加入 氧化剂(液氯、次氯酸钠、二氧化氯和臭氧等)法、防 污橡胶、化学防污涂料法和电解防污法等. 其中涂装 防污涂料法具有操作简单、效果明显且应用范围广泛 等优点, 因此成为目前最常用的防污方法之一.

最初的防污涂料技术可以追溯到 1625 年 $^{[7]}$, 但 是直到 19 世纪 60 年代才有可实用的防污涂料出现, 其中实际有效的是以砷、铜和录化合物为毒剂, 树脂 为基料的热塑型和冷塑型漆(图 2). 20 世纪以来, 防 污涂料的研究迅速发展, 但直至 1970 年以前, 铜和 铜化合物(主要是氧化亚铜)仍一直是防污漆的主要 防污剂, 有时也会配以氧化录和有机金属化合物如 铅、录、砷、锌、锡等防污剂. 20 世纪 60 年代中期, 以 有机锡高聚物 (tributyltin, TBT) 为代表的自抛光型防 污涂料的发明, 标志着防污技术的新高度, 其广泛应 用为航运事业带来了巨大的经济利益. 这些防污涂 料虽然从一定程度上都有效地抑制污损生物的附着, 但其中的有毒成分不断向海水释放, 对渔业和海洋 环境造成了极大的危害 ${ }^{[8]}$. 因此, 国际海事组织决议 规定, 2003 年 1 月 1 日开始禁止生产 TBT 防污涂料, 2008 年 1 月 1 日开始禁止使用 TBT 防污涂料 ${ }^{[9]}$. 目 前虽然以重金属和生物杀虫剂为抗污成分的防污漆 作为 TBT 替代品被广泛使用, 但它们对非目标海洋 生物的毒性依然不容忽视, 越来越多的国家开始禁 止使用以有机金属为主要抗污成分的涂料, 因此寻 找一种有效的对环境无害的防污涂料或开发一种新 型的环保型防污技术是一项迫在眉睫的科学难题.

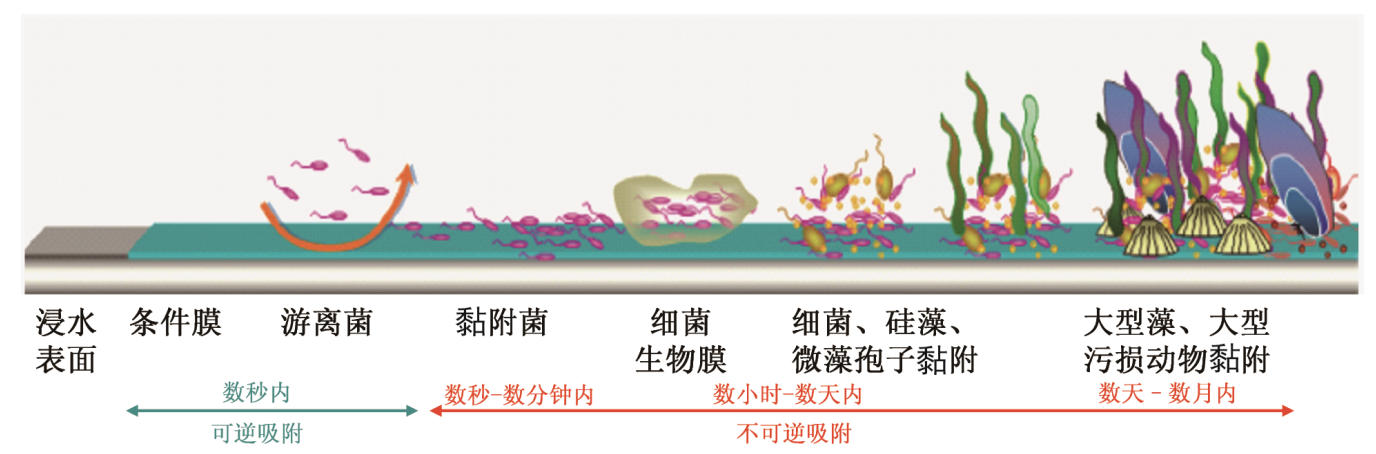

图 1 海洋生物污损形成过程(网络版彩图)

1080 


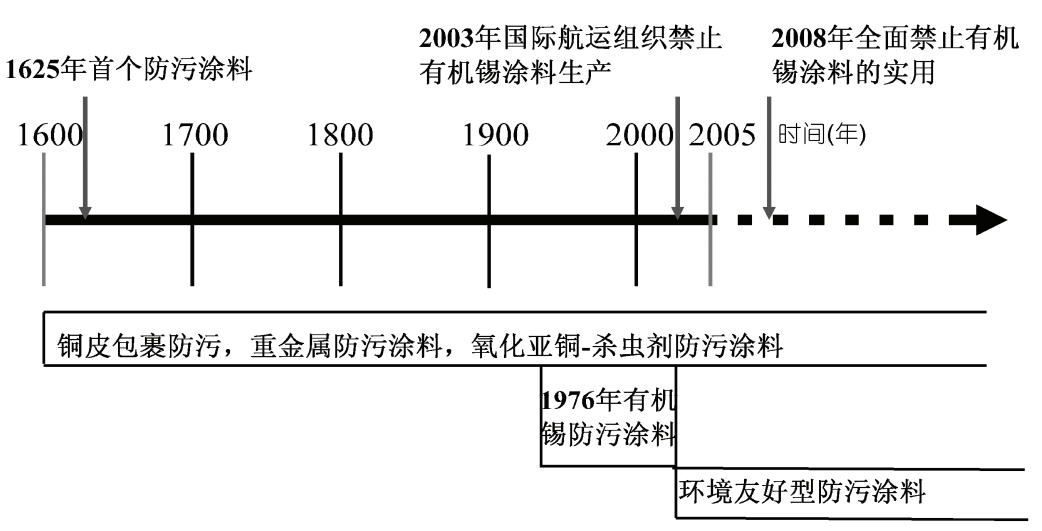

图 2 防污剂的发展历程

\section{3 化学生态学与污损生物防除}

化学生态学属于生态学的分支, 是化学和生态 学的交叉学科, 是研究生物与环境以及生物间的(化 学)联系规律及其机制, 并将学科原理加以应用的科 学 ${ }^{[10]}$. 化学生态学是近年来发展起来的一门交叉学 科, 它通过对介入生物群体内或介入生物群体间的 天然化学物质的起源、功能以及重要性进行研究, 以 求得对宏观生态现象的透彻理解 ${ }^{[11]}$. 研究内容涉及 生物适应环境或生物间相互适应的化学机制, 生物 精确利用信息化学物质, 生物间通过次生化学物质 为媒介进行相互联系, 及生物对化学物质的反应等. 在海洋生态系统中, 化学生态学的研究还处于起步 阶段, 真正了解并将之用于实践, 还需要科研工作者 长期的共同努力.

海洋污损生物群落以复杂的生物、化学和物理的 相互作用为特征, 具有动态性、非均一性和非连续性 等特性. 化学生态学应用于海洋污损生物的研究, 将 有利于阐明控制生物附着机理和了解生物衍生的外 聚合物与附着表面的化学和物理的相互作用, 并促 进解决生物污损这一难题的新思路和新方法的产生. 随着海洋化学生态学研究的加深, 基于对海洋环境 的生态安全问题考虑, 利用海洋生物的天然防污本 领开发新型、高效、环保的防污技术已成必然.

许多海洋生物的体表能保持光滑不被污损生物 附着, 显示出很强的防污能力, 这表明它们在长期进 化过程中建立了一系列的防御策略, 包括化学防污 机制(防污活性分子、生物酶、表皮黏液、双电性等)、 物理防污机制(微观结构、表面能等)、忍耐和躲避作
用机制(通过蜕皮、跃出水面、自身清洗等生活习性

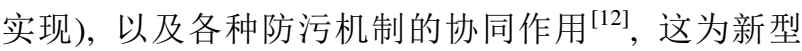
环保防污技术的开发提供了仿生学对象, 为仿生防 污材料的开发提供了重要依据. 目前仿生防污材料 的研究和开发主要集中于两种思路：（ｉ）寻找并利 用合适的生物防污剂, 在不破坏环境及其他非目标 生物的前提下防止污损生物附着; (ii) 通过设计特 殊的表面和本体材料特性来模仿具有防污功能的生 物特征，使浸水材料表面附着力尽可能降到最低，从 而使之不易被污损生物附着, 最终达到防止海洋污 损的目的.

生物防污剂是指生物体基于化学防御和自身净 化的化学生态学作用而表现出抗污活性的天然产 物 ${ }^{[13]}$, 主要是生物体的次级代谢物、蛋白酶等具有一 定活性和功能的成分. 目前已报道的抗污活性天然 产物主要来源于海洋微生物、海藻、海洋无脊椎动物 (如海绵), 还有一部分来源于陆地生物. 海洋微生物 生长速度快且容易大规模培养, 能够快速量产防污 活性物质，自身也可以直接作防污剂添加到涂料中， 是生物防污剂的重要来源. 目前已经从海洋微生物 中获得大概 20 多个抗生物污损天然产物, 分别有脂 肪酸类、异丁烯内酯类、环二肽类、二羟基苯酸大环 内酯类等 ${ }^{[13]}$. 海洋藻类、无脊椎动物和陆地植物也是 生物防污剂的重要来源. 从红藻中分离得到的卤代 呋喃酮化合物、绿藻提取的萜类、大叶藻中分离的 $\mathrm{p}$-肉桂酸硫酸酯、海绵中提取的吡喃、甾体硫酸盐、 嘌呤碱、酰胺及呋喃萜类、珊瑚中分离获得的二萜类 物质和开环甾族化合物、陆生植物特别是中药材中分 离提取的丹皮酚、辣椒素、千金子甾醇、齐墩果酸、 
香豆素内酯等均具有一定的抗污活性 ${ }^{[14 \sim 18]}$. 以海洋 中天然活性物质提取物为防污剂, 最成功也最有使 用价值的是 Sea-nine $211^{[19]}$ (图 3), 其中的噻唑啉酮结 构就是一种生物防污剂经过结构改造而得到的, 其 开发者美国罗门哈斯公司也因此获得了“总统绿色化 学挑战奖”.

从陆生植物的叶面到海洋软体动物的甲壳以及 大型海洋哺乳动物鲨鱼和海豚的表皮, 都有着复杂 的表面形貌结构, 这些生物表面的形貌结构能够阻 止或抑制生物附着. 根据鲨鱼防护海底生物附着的 机理, Brennan 等人 ${ }^{[20]} 、$ Scardino 和 De Nys ${ }^{[21]}$ 及 Magin 等人 ${ }^{[22]}$ 利用刻蚀翻模的方法研制出 Sharklet AFTM 微结构材料(图 4), 可以有效地防止藻类、藤壶等污 损海生物的附着, 使其附着率降低 85\%左右. 由欧洲 联盟资助, 12 个国家 31 个研究单位共同参与的 Ambio (人类环境杂志) 项目对微/纳米防污结构进行了 研究 ${ }^{[23]}$, 如基于多壁碳纳米管、海泡石构建纳米微结 构、基于氟硅共聚材料通过微相分离制备微结构等, 实验表明这些微/纳米结构能够防止藻类和藤壶的 附着.

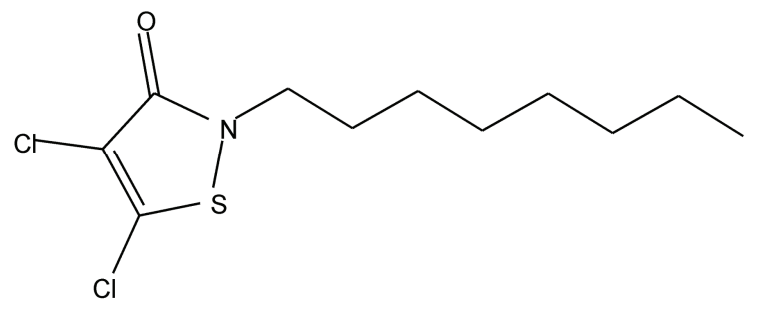

图 3 Sea-nine 211 的结构

\section{4 结语}

用化学生态学方法寻找天然防污剂或开发新型 防污材料扩展了海洋污损防除研究的思路, 适应了 目前保护环境, 保护良好生态系统的环保理念. 这方 面研究的开展有利于高效无毒污损防除产品的开发, 并有利于促进对海洋生物群落和生态系统结构本质 及生物间相互作用关系的深入认识. 同时, 必须认识 到生物的防污功能是多个因素通过耦合、协同作用的 结果, 这涉及多门学科的交叉, 因此需要加强基础研 究与应用研究的结合和转化, 并促进化学、生物学、 生态学、环境科学等各门学科在海洋污损生物研究中 的应用与合作.

本实验室针对海洋中生物污损问题, 认识生物 污损形成过程中微型生物污损的形成及作用; 根据 众多生物体表不易被污着的宏观生态特性和化学生 态学原理, 研究其抗污着的化学和物理机制, 篮选和 开发环境友好防污剂和长效防污涂层用于污损生物 防除. 在污损形成及防污机制的研究方面, 发现污损 生物的附着呈由小到大的趋势, 且微型生物污损群 落包括芽孢杆菌、假交替单胞菌等 110 多种微生物和 菱形藻、舟形藻、筒柱藻、卵形藻、曲壳藻等硅藻, 并 且呈现出一定的季节变化规律 ${ }^{[24,25]}$; 此外还发现介 导微生物群体感应的二酮哌嗪类和高丝氨酸内酯类 信号分子(结构见图 5)能够调控污损生物的附着 ${ }^{[25,26]}$. 在生物源天然防污剂的研究与开发方面, 研究发现, 生物源吲哚类物质、辣素(结构见图 5)等 ${ }^{[25 ~ 32]}$ 具有良 好的抗污活性, 海上挂板半年能显著抑制大型污损 生物的附着; 此外还从海岸带耐盐植物中分离

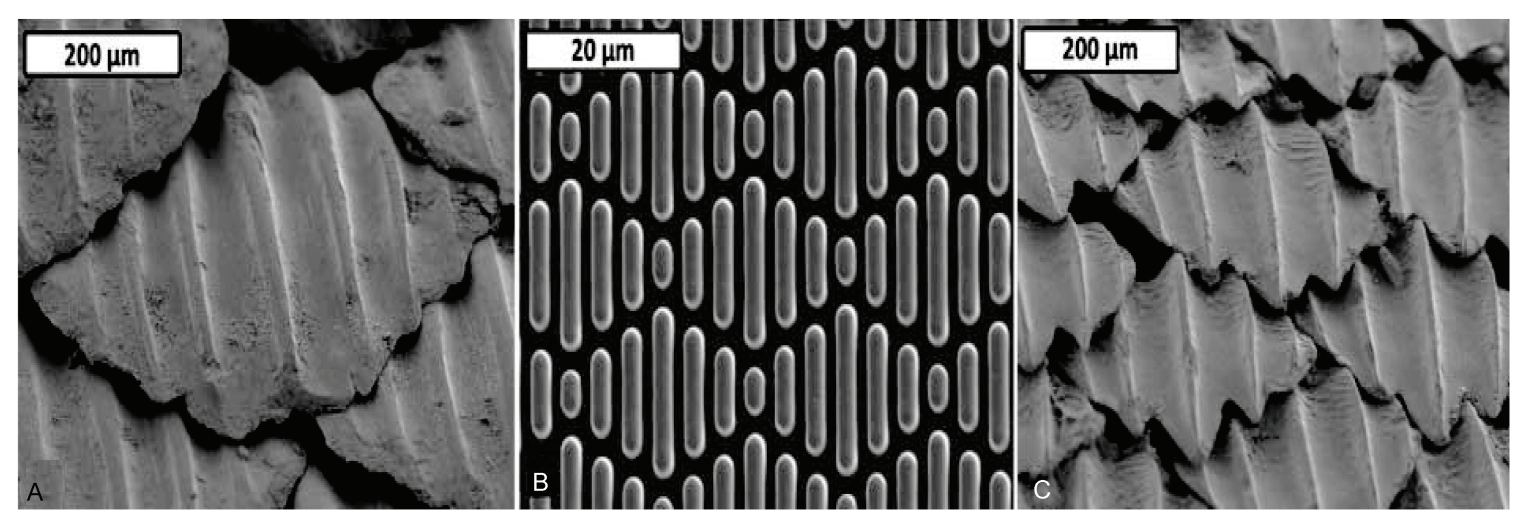

图 4 鉴鱼表皮的微结构 $(\mathrm{A}, \mathrm{C})$ 及其仿生材料表面 $(\mathrm{B})^{[21]}$ 
<smiles>CC(C)CC1NC(=O)C2CCCN2C1=O</smiles><smiles>[R]C([R])CC(=O)NC1CCOC1=O</smiles>

AHL $\left(\mathbf{R}_{1}=\mathbf{O}\right.$, H or $\mathrm{OH}$; $\left.\mathbf{R}_{2}=\mathrm{C}_{\mathrm{n}} \mathrm{H}_{2 n+1}\right)$<smiles>[X]c1ccc2cc[nH]c2c1</smiles>

吲哚 $(\mathrm{X}=\mathrm{Cl}$ or $\mathrm{Br})$<smiles>CCCCCCCCC(=O)NCc1ccc(O)c(OC)c1</smiles>

辣素<smiles>COc1ccc(-c2coc3c(OC)c(O)ccc3c2=O)cc1</smiles>

黄酮类物质(分离自罗布麻)

图 5 本实验室发现的各种防污化合物

得到多种抗污活性先导化合物(结构见图 5) ${ }^{[33]}$, 为生 物源防污剂的篮选提供了物质基础. 在仿生防污纳 米涂料的研究与开发方面, 经过化学改性的纳米 $\mathrm{TiO}_{2}$ 其分散性与稳定性得到显著提高 ${ }^{[34]}$, 将其与丙
烯酸树脂复合制备成防污涂料，具有底表面能的特 性, 且抗污活性有了较大提高, 海上挂板半年基本无 大型污损生物附着. 这些研究为海洋生物污损环境 友好防除提供了技术和方法支持.

\section{参考文献}

1 Callow M E, Callow J A. Marine biofouling: a sticky problem. Biologist, 2002, 49: 1-5

2 黄宗国. 海洋污损生物及其防除. 北京: 海洋出版社, 1984

3 Yebra D. Antifouling technology-past, present and future steps towards efficient and environmentally friendly antifouling coatings. Prog Org Coat, 2004, 50: 75-104

4 Schultz M P, Bendick J A, Holm E R, et al. Economic impact of biofouling on a naval surface ship. Biofouling, 2011, 27: 87-98

5 Horne R A. Marine Chemistry: the Structure of Water and Chemistry of the Hydrosphere. New York: Wiley-Interscience, 1969. 568

6 Alberte R S, Snyder S, Zahuranec B J. Biofouling research needs for the United States Navy: programs history and goals. Biofouling, 1992, 6: 91-95

7 Woods Hole Oceanographic Institution. Marine Fouling and its Prevention. Annapolis: US Naval Institute Press, 1952

8 Dafforn K A, Lewis J A, Johnston E L. Antifouling strategies: history and regulation, ecological impacts and mitigation. Mar Pollut Bull, 2011, 62: 453-465

9 Champ M A. The status of the reaty to ban TBT in marine antifouling paints and alternatives. In Proceedings of the $24^{\text {th }}$ (US/Japan) Marine Faci lities Panel Meeting, Hawaii, 2001

10 间风鸣. 化学生态学. 北京: 科学出版社, 2003

11 方芳, 严涛, 刘庆. 化学生态学在海洋污损生物防除中的应用. 应用生态学报, 2005, 16: 1997-2002

12 Ralston E, Swain G. Bioinspiration-the solution for biofouling Control. Bioinsp Biomim, 2009, 4: 1-9

13 钱培元. 抗污损海洋天然产物的开发及其作用机理研究进展. 生命科学, 2012, 24: 1026-1034

14 Kjelleberg S, Steinberg P. Surface warfare in the sea. Microbiol Today, 2001, 28: 134-135

15 Smyeniotopoulos V, Adbatis D, Tziveleka L A, et al. Acetylene sesquiterpeniod esters from the green alga Caulerpa prolifera. J Nat Prod, 2003, 66: 21-24

16 Todd J S, Zimmerman R C, Crews P, et al. The antifouling activity of natural and synthetic acid sulphate eaters. Phytochemistry, 1993, 34: 401-404

17 Yang L H, Lee O O, Jin T, et al. Antifouling properties of 10 beta-formamidokalihinol-A and kalihinol A isolated from the marine sponge Acanthella Cavernosa. Biofouling, 2006, 22: 23-32

18 Tomono Y, Hirota H, Fusetani N. Isogosterones A-D, Antifouling 13,17-secosteroids from an octocoral Dendronephthya sp. J Org Chem, 
1999, 64: 2272-2275

19 Braithwaite R A, Fletcher R L. The toxicity of Irgarol 1051 and Sea-Nine 211 to the non-target macroalga fucus serratus Linnaeus, with the aid of an image capture and analysis system. J Exp Mar Bio Ecol, 2005, 322: 111-121.

20 Brennan A B, Baney R H, Carman M L. Surface topography for non-toxic bioadhesion control. US Patent, 7143709, 2006-12-05

21 Scardino A J, De Nys R. Biomimetic models and bioinspired surfaces for fouling control. Biofouling, 2011, 27: 73-86

22 Magin M M, Long C J, Cooper S P, et al. Engineered antifouling microtopographies: the role of reynolds number in a model that predicts attachment of zoospores of Ulva and cells of Cobetia marina. Biofouling, 2010, 26: 719-727

23 Rosenhahn A, Ederth T, Pettitt M E. Advanced nanostructures for the control of biofouling: the FP6 Integrated Project AMBIO. Biointerphases, 2008, 3: 1-5

24 Yang C Y, Wang J H, Yu Y, et al. Seasonal variation of fouling diatom community on Yantai coast. Chin Oceanol Limnol, 2015, 33: 439-446

25 于洋, 王建华, 方圣涛, 等. 海洋污损细菌群落结构及其发酵液中化学成分的研究. 海洋科学, 2014, 9: 27-32

26 于洋, 王建华, 方圣涛, 等. 污损生物膜细菌 Pseudoalteromonas issachenkonii YT1305-1 的鉴定及其代谢物化学成分分析. 微生物 学通报, 2014, 41: 1278-1286

27 Yang C Y, Yu Y N, Sun W J, et al. Indole derivatives inhibited the formation of bacterial biofilm and modulated Ca ${ }^{2+}$ efflux in diatom. Mar Pollut Bull, 2014, 88: 62-69

28 Yang C Y, Sun W J, Liu S J, et al. Comparative effects of indole derivatives as antifouling agents on the growth of two marine diatom species. Chem Ecol, 2015, 31: 299-307

29 Chen J C, Zhang Z K, Liu S J, et al. One-pot tandem synthesis of 2,3-unsubstituted indoles, an improved Leimgruber-Batchoindole synthesis. RSC Adv, 2014, 4: 4672-4675

30 Yang C Y, Zhou J, Liu S J, et al. Allelochemical induces growth and photosynthesis inhibition, oxidative damage in marine diatom Phaeodactylum tricornutum. J Exp Mar Biol Ecol, 2013, 444: 16-23

31 Yang C Y, Liu S J, Ma X X, et al. Effects of ethyl 2-methyl acetoacetate (EMA) on the growth of Phaeodactylum tricornutum and Skeletonema costatum. Chem Ecol, 2012, 28: 524-534

32 Zhou J, Yang C Y, Wang J H, et al. Toxic effects of environment-friendly antifoulant nonivamide on Phaeodactylum tricornutum. Environ Toxicol Chem, 2013, 32: 802-809

33 Kong N N, Fang S T, Liu Y, et al. Flavonoids from the halophyte Apocynum venetum and their antifouling activities against marine biofilm-derived bacteria. Nat Prod Res, 2014, 28: 928-931

34 孙秀敏, 徐金光, 刘苏静, 等. 纳米 $\mathrm{TiO}_{2}$ 的改性及其性能研究. 化工新型材料, 2014, 42: 130-133

\title{
Novel Antifouling Technology Research: Progress and Prospects
}

\author{
WANG JianHua, YANG CuiYun, LIU SuJing, FANG ShengTao \& XIA ChuanHai \\ Key Laboratory of Coastal Biology and Biological Resources Utilization, Yantai Institute of Coastal Zone Research, Chinese Academy of Sciences, \\ Yantai 264003, China
}

Marine biofouling has brought about detrimental costs to maritime activities, and the traditional antifouling technology has been increasingly ineffectual towards meeting the requirements of environment. Research and development of new, environment-friendly antifouling agents is therefore imminent. Development of new, non-toxic antifouling materials and technology based on bionics and chemical ecology is a novel idea to solve the problem of marine fouling. This paper reviews the advances in antifouling technology, and introduces bionic antifouling compounds and materials based on chemical ecology. It also proposes prospects for further development of bionic antifouling technology.

\section{biofouling, antifoulant, chemical ecology, bionic antifouling technology}

doi: 10.1360/N052016-00065 